\section{PATTERN RECOGNITION}

\section{Legs and Beaks}

from our Animal Behaviour Correspondent THE problem of how animals recognize patterns of stimulation from their environments is complex and difficult, particularly where apparently similar stimuli elicit two different responses. Finding the physiological basis for such pattern recognition is of considerable interest. Electro-physiological investigations of the visual system of the cat by D. H. Hubel and T. N. Wiesel (J. Physiol., 148, 574; 1959) revealed the presence of units specifically responsive to vertical dark bars. Hubel and Wiesel subsequently found (J. Neurophysiol., 28, 229; 1965) other cells in the cortex of the cat which respond to a slit or bar, but only if it is of limited length. These they called "lower order hypercomplex" cells. Recently, J. P. Hailman has used behavioural evidence to infer that the visual system of a gull chick contains units at least as highly organized as these hypercomplex cells (Anim. Behav., 19, 328; 1971).

A newly-hatched laughing gull chick (Larus atricilla) will peck at its parent's down-pointing, reddish beak and this induces the parent to regurgitate food. In spite of the fact that the legs of the parent gull provide all the known relevant stimulus characteristics (size, shape, colour, orientation) that elicit the beak pecking response, even newly hatched chicks are able to discriminate legs from beak. They only rarely peck at their parent's legs.

Hailman found that a rod or stripe held vertically in front of the chick so that it projected from above and stopped in the centre of the chick's visual field (as would a beak) would be readily pecked by a chick. A chick was much less likely to peck at a rod or stripe projecting vertically upwards from the floor and ending in the centre of the visual field (about where the legs would join the parent's body). This provided a clue as to how the chick was discriminating between leg and beak.

Hailman at first thought that the visual system of the chick contained nothing more complicated than units detecting vertical bars, that is, units which respond to the presence of vertical bars however long or short the bars might be. If more of these units were connected to the upper half of the retina than were connected to the lower half, this would explain why the "beak" stimulus was more attractive than the "leg" stimulus, as the rod pointing downwards from above would excite more of these units than the rod pointing upwards from below. But a long vertical stripe which cut across the whole of the chick's visual field ought, in these circumstances, to be an even better stimulus, as it would stimulate more of the retina than either of the half-bars.

This expectation was not confirmed. The full-stripe stimulus elicited less pecking than the downward pointing half-stripe (the "beak"). This means that there must be some cells which integrate information from different parts of the retina and respond only when there is a dark vertical bar in the upper portion of the retina and not such a bar in the lower half. They detect not just vertical bars in certain places, but their absence from other positions as well. Such an operation is similar in complexity to that performed by the cat's cortical cells described by Hubel and Wiesel. The search for the units responsible for the gull chick's perception can begin in the knowledge that the perception is organized in a fashion at least as complex as the hypercomplex units of the cat.

\section{LIPID MEMBRANES \\ Nervous Hysteresis}

from our Membrane Correspondent

MORE than thirty years ago the lipid bilayer was put forward by Danielli as an important structural element in biological membranes. Interest in the role of membrane lipids has since fluctuated but is currently very strong and one of the recent areas of activity concerns the role of lipid phase transitions.

In the past few years a battery of physical methods (X-ray diffraction, nuclear magnetic resonance, differential thermal analysis and the like) has been brought to bear on the subject of phase transitions in phospholipid-water systems and most of the emphasis has been on the so-called melting of the phospholipid hydrocarbon chains in lamellar lipid arrays (bilayers). Over some narrow temperature range characteristic of the particular phospholipid there is usually a transition in the configuration of the hydrocarbon chains from a relatively rigid structure, like that in solid hydrocarbons, to a much more fluid state. This fluid state presents less of a barrier to permeating molecules and ions and is thus the state in which the lipid bilayers would be expected in functioning cell membranes. This expectation was borne out in some recent work of G. B. Ashe and J. M. Steim (Biochim. Biophys. Acta, 233, 810 ; 1971), who showed that for growth of bacteria to occur it was necessary for the membrane lipids to be in this fluid state.

Attached to their hydrocarbon tails phospholipid molecules also, of course, have hydrophilic head groups. How these head groups might behave at transition temperatures has often been only a matter of passing comment but they evidently also undergo some change in spacing and configuration. How they might do this and its implications in biological membranes is the subject of a recent report by $\mathrm{H}$. Träuble (Naturwissenschaften, 58, 277 ; 1971).

Träuble used the popular fluorescent probe 1-analino-8-naphthalene sulphonate (ANS) to investigate the model membrane system in aqueous dispersions of dipalmitoyl phosphatidyl choline (lecithin) which undergo a phase change in the temperature range $40-46^{\circ} \mathrm{C}$. Träuble showed that the ANS was intercalated between the head groups at the surface of the lecithin bilayers and the increase in fluorescence which he

\title{
Cellular Ribosomes in Arenoviruses
}

THERE are so many well documented examples of viruses which contain cellular enzymes and/or nucleic acids that Pedersen's claim to have detected ribosomes in lymphocytic choriomeningitis virus particles is not perhaps all that surprising. Electron micrographs of this virus and its relatives reveal within the virions sand-like or ribosome-like granules, from which the group gets its name, the arenoviruses. Pedersen, as he reports in Nature New Biology next Wednesday, set about characterizing the RNA species in lymphocytic choriomeningitis virus (LCM virus) in an attempt to establish that these granules are ribosomes. His results substantiate this idea.

The viral RNA is resolved by gel electrophoresis into four components, two of which co-electrophorese with the two cellular ribosomal RNAs. Viruses grown in cells exposed to low doses of actinomycin $\mathrm{D}$, which inhibit ribosomal
RNA synthesis, lack these two species of RNA. The two remaining species, presumably authentic LCM virus genomic RNAs, sediment in sucrose gradients at $23 \mathrm{~S}$ and $31 \mathrm{~S}$ and probably have molecular weights of about $1.1 \times$ $10^{6}$ and $2.1 \times 10^{6}$ respectively.

Because the two putative ribosomal RNAs in LCM virus particles account for about 50 per cent of the total RNA and because treatment of the intact virions with ribonuclease does not eliminate these two RNA species, Pedersen is confident that they are not simply contaminants adsorbed to the surface of the virus particles. In short, the granules seen in electron micrographs of the arenoviruses are almost certainly ribosomes which are presumably engulfed into the virions as they bud from the surface of infected cells, electron micrographs of which reveal accumulations of ribosomes at the site of progeny virus formation. 